\title{
Seasonal and spatial variability of carbon monoxide (CO) in the Martian atmosphere from PFS/MEX observations
}

\author{
Jimmy Bouche ${ }^{1}$,Pierre-François Coheur ${ }^{1}$, Marco Giuranna ${ }^{2}$, Paulina \\ Wolkenberg ${ }^{2}$, Luca Nardi ${ }^{3,2}$, Marilena Amoroso ${ }^{4}$, Ann Carine Vandaele ${ }^{5}$, \\ Frank Daerden ${ }^{5}$, Lori Neary ${ }^{5}$, Sophie Bauduin ${ }^{1}$
${ }^{1}$ Université libre de Bruxelles (ULB), Spectroscopy, Quantum Chemistry and Atmospheric Remote Sensing (SQUARES) CP 160/09, Avenue F.D. Roosevelt 50, Bruxelles, Belgium.
${ }^{2}$ National Institute of Astrophysics INAF-IAPS, Rome, Italy. 3 University of Rome "La Sapienza", Rome, Italy.
${ }^{4}$ Agenzia Spaziale Italiana, Sede di Matera, Italy.
${ }^{5}$ Royal Belgian Institute for Space Aeronomy, Brussels, Belgium.

Key Points:

- PFS observations are used to investigate the global distribution and the seasonal cycle of $\mathrm{CO}$ on Mars in weak dust conditions

- The seasonal cycle is driven by the sublimation/condensation of $\mathrm{CO}_{2}$, with a large variability around a mean CO VMR of about $820 \mathrm{ppm}$

- The distribution and temporal evolution agree generally well with previous observations and with simulations from the GEM-Mars model

Corresponding author: Jimmy Bouche, jbouche@ulb.ac.be 


\begin{abstract}
A subset of more than 100000 nadir measurements covering more than 7 Martian years (MY 26 to MY 33) recorded in the thermal part of the Short Wavelength Channel (SWC) from the Planetary Fourier Spectrometer (PFS) on board Mars Express is exploited to investigate the global distribution and the seasonal cycle of carbon monoxide $(\mathrm{CO})$ on Mars. The retrieval of $\mathrm{CO}$ vertical profiles is successfully achieved using a methodology based on the optimal estimation but the low information content is such that we mainly discuss the variability in $\mathrm{CO}$ in terms of integrated columns (from the surface to $24 \mathrm{~km}$ ) or the associated column-averaged mixing ratio. We find a strong seasonality in $\mathrm{CO}$, especially at mid and high-latitudes, which confirms earlier work and the current knowledge of the $\mathrm{CO}_{2}$ condensation/sublimation cycles, as implemented for instance in the Global Environmental Multiscale (GEM) general circulation model for Mars, that we use as a basis for comparison. We report a general consistency between model and observation, with a tendency of the latter to provide lower $\mathrm{CO}$ volume mixing ratios (VMRs), except at low latitudes. The spatial distribution of the CO column-averaged VMR is obtained on a seasonal basis and investigated in terms of large-scale patterns but also local peculiarities. Finally, we show that the retrieved profiles systematically present strong $\mathrm{CO}$ vertical gradients close to the surface in mid- and equatorial latitudes, likely related to the vertical sensitivity of PFS rather than real near-surface $\mathrm{CO}$ enrichment.
\end{abstract}

\title{
Plain Language Summary
}

The atmosphere of Mars is mainly composed of carbon dioxide ( 95\%) and other carbon compounds in much lower concentration, including carbon monoxide. The relative abundances of the carbon compounds depend on each other and they vary during a Martian year primarily according to the temperature cycle. In the colder periods carbon dioxide indeed freezes (mainly at the polar caps), while it sublimates under higher temperatures, causing large variations to atmospheric pressure and the volume mixing ratio of the trace species. This study examines the variability of carbon monoxide in the low atmosphere of Mars (from the surface to $24 \mathrm{~km}$ ), on a global scale. The CO seasonal cycle is obtained by exploiting $\sim 100000$ observations made by the Planetary Fourier Spectrometer on-board the Mars Express satellite in almost aerosol-free conditions. For each individual Planetary Fourier Spectrometer observation, a CO vertical profile is retrieved. The resulting column distributions and time evolutions, showing large variability especially at mid and high latitudes, are in good agreement with previous work as well as with simulated distributions from a state-of-the-art chemistry-transport model.

\section{Introduction}

The satellite observations made in the last 20 years have allowed to better represent the spatial, vertical and temporal variability of CO. Infrared observations of the 1-0 and 2-0 bands of CO (centered at 2143 and $4235 \mathrm{~cm}^{-1}$, respectively), performed from the ground (Billebaud et al., 1992, 1998; Krasnopolsky, 2003; Clancy et al., 1990; Lellouch et al., 1991) or from space using the OMEGA (Encrenaz et al., 2006) and PFS instruments (Billebaud et al., 2009; Sindoni et al., 2011) on-board Mars Express (MEX) and CRISM (M. D. Smith et al., 2009, 2018; Holmes et al., 2019) on-board the Mars Reconnaissance Orbiter (MRO), have in particular contributed to this. From those it became clear that $\mathrm{CO}$ is not uniformly mixed and presents latitudinal gradients and seasonal variations, especially at high latitudes. These variations are intimately related to the condensation-sublimation cycle of $\mathrm{CO}_{2}$ at the poles, which creates an enhancement of non-condensable gases, including $\mathrm{CO}$, during the respective winters. The first evidence of these processes came from ground-based observations (Krasnopolsky, 2003), that showed a North-to-South increase of the CO VMR during the Northern summer (due to the condensation of $\mathrm{CO}_{2}$ at the South polar cap). A more complete picture of the latitudinal-seasonal changes of CO VMR is now available 
thanks to the measurements of instruments on-board spacecraft (OMEGA, PFS, CRISM), that led to distributions in line with the general circulation model $(\mathrm{GCM})$ simulations for non-condensable species (Encrenaz et al., 2006; Forget et al., 2008; Lian et al., 2012). A more recent distribution from CRISM is reported by M. D. Smith et al. (2018), who improved on the earlier work (M. D. Smith et al., 2009) by upgrading the radiative transfer modeling. This work reported distributions of $\mathrm{CO}$ total columns and also used the GEMMars GCM developed Neary and Daerden (2018) and Daerden et al. (2019) as a basis of comparison.

Retrievals of weakly resolved CO vertical profiles in the Martian atmosphere were made by Bouche et al. (2019) from nadir observations of PFS on-board MEX spacecraft. Those provide CO abundances essentially in the lower atmosphere $(<20 \mathrm{~km})$ and offer thereby the possibility to complement the much better resolved profiles that will become available at higher altitudes from the occultation sounder (NOMAD) (Vandaele et al., 2018) on the ExoMars Trace Gas Orbiter (TGO) spacecraft. Recent model distributions of CO were also obtained by data assimilation Holmes et al. (2019), forcing a GCM model with CRISM total columns (M. D. Smith et al., 2018) and Mars Climate Sounder (MCS) temperature profiles (Kleinböhl et al., 2017). These previous works tend to show that CO profiles present vertical gradients in some regions and at certain seasons, but mostly in the middle/upper atmosphere.

This work adapts and extends the exploratory work of Bouche et al. (2019) for the retrieval of $\mathrm{CO}$ vertical profiles in the lower Martian atmosphere from observations of PFS/MEX. It exploits a much larger sample of individual spectra spanning 7 years of PFS observations, in the objective to reveal the spatial and temporal variations of $\mathrm{CO}$ in the lowest layers. Section 2 presents the retrieval method, the criteria used to build a large set of clear spectra and the main retrieval parameters. In Section 3 the results are presented and discussed, starting with the seasonal variation of retrieved CO VMR, adding a comparison against those simulated with the GEM-Mars GCM model developed by Neary and Daerden (2018) (additional details in Daerden et al. (2019)). The spatial variation of the retrieved $\mathrm{CO}$, in terms of the $\mathrm{CO}$ total column and its vertical profile are presented and discussed to complete Section 3. The conclusions of the study are summarized in Section 4.

\section{CO retrievals}

This section recalls the main steps of the methodology used to retrieve $\mathrm{CO}$ profiles from PFS observations, in the 1-0 band. A complete description of the PFS instrument can be found in Formisano et al. (2005) and Giuranna et al. (2005). The retrieval methodology, which uses the Optimal Estimation Method (OEM) (Rodgers, 2000), was presented in Bouche et al. (2019). In that study only a small number of PFS spectra were analysed and used to characterize the $\mathrm{CO}$ retrievals in terms of errors and vertical sensitivity. In particular we found that the PFS measurements were sensitive to the $\mathrm{CO}$ abundance in the atmosphere below $15 \mathrm{~km}$ but that, unfortunately, they do not allow to retrieve independently the $\mathrm{CO}$ abundance at different altitudes in this lower part of the atmosphere. The integrated $\mathrm{CO}$ column is therefore the most representative quantity of the information contained in the nadir PFS spectra and the resulting 0-15 km column was retrieved with a total error between 5 and $15 \%$. Only measurements in daylight were found to yield sufficient signal to retrieve $\mathrm{CO}$ from single PFS observations.

Following this initial study, we similarly use in this work the Atmosphit software, in which OEM is implemented, to retrieve CO profiles from a large set of individual PFS spectra spanning 7 Martian years. Atmosphit is a versatile line-by-line radiative transfer code developed at the Université libre de Bruxelles (ULB), which, until recently, was mainly used for the Earth's atmosphere (e.g. Barret et al. (2005)). 
The objective of this work is to retrieve $\mathrm{CO}$ profiles/columns from a sufficiently large set of PFS observations to draw (quasi) global distributions and seasonal variations. The selection of spectra was made from part of the PFS measurements dataset, spanning the period from MY 27 to MY 33. This corresponds to more than 1 million measurements. The selection criteria are similar to those used in our initial work (Bouche et al., 2019), as summarized in Table 1. In order to gather sufficient measurements, the criteria on the absolute radiance (at $\left.2165 \mathrm{~cm}^{-1}\right)$ and on the ice and dust opacities $\left(\tau_{\mathrm{i}}\right.$ and $\tau_{\mathrm{d}}$ respectively) have, however, been slightly relaxed. The values of $\tau_{\mathrm{d}}, \tau_{\mathrm{i}}$ and of surface temperature $\left(\mathrm{T}_{\mathrm{s}}\right)$ were retrieved from the PFS Long Wavelength Channel (LWC) measurements independently (Wolkenberg et al., 2018; Giuranna et al., 2019).

Table 1. Criteria set for selecting PFS spectra in this work, compared to Bouche et al. (2019). $I_{2165}$ stands for the value of the radiance at $2165 \mathrm{~cm}^{-1} . \Delta \mathrm{BT}$ is the brightness temperature difference between a CO line at $2165.7 \mathrm{~cm}^{-1}$ and two baseline channels (see Bouche et al. (2019) for details); $\tau_{\mathrm{d}}$ and $\tau_{\mathrm{i}}$ are the dust and ice integrated opacities (Wolkenberg et al., 2018; Giuranna et al., 2019); $\mathrm{T}_{\mathrm{s}}$ is the surface temperature (Wolkenberg et al., 2018; Giuranna et al., 2019).

\begin{tabular}{ccc}
\hline Criteria & Bouche et al. (2019) & This work \\
\hline$\tau_{\mathrm{d}}$ & ] $0-0.15[$ & ] $0-0.2[$ \\
$\tau_{\mathrm{i}}$ & ] $0-0.15[$ & ] $0-1]$ \\
$I_{2165}\left(\mathrm{erg} \mathrm{s}^{-1} \mathrm{sr}^{-1} \mathrm{~cm}^{-2} \mathrm{~cm}\right)$ & $0.025<I_{2165}$ & $0.4<I_{2165}$ \\
$\Delta \mathrm{BT}(\mathrm{K})$ & $2.2<\Delta \mathrm{BT}$ & $2.2<\Delta \mathrm{BT}$ \\
$\mathrm{T}_{\mathrm{s}}(\mathrm{K})$ & $240<\mathrm{T}_{\mathrm{s}}$ & $240<\mathrm{T}_{\mathrm{s}}$ \\
Time & daytime & daytime \\
\hline
\end{tabular}

A total of around 108.000 spectra were kept with these selection criteria. Note that by a way of consequence these are only "clear" or almost clear scenes during daylight (all nighttime measurements are rejected). This also means that the polar winters are not covered and excluded from our analyses.

The choice of spectral window, retrieval settings and input parameters to the radiative transfer is also given in Bouche et al. (2019). The only changes with respect to this initial work concern the vertical extent of the atmosphere, which is limited to $30 \mathrm{~km}$ here (it was $60 \mathrm{~km}$ in Bouche et al. (2019)) without modifying the quality of the final fitted spectrum, and the fact that a total column of $\mathrm{CO}_{2}$ is fitted instead of a full profile. It is also important to note that instead of retrieving first for each spectrum an effective reflectivity by using a large spectral range $\left(2040-2600 \mathrm{~cm}^{-1}\right)$, we proceed here in two steps: first we estimate the effective reflectivity by using a relation (obtained on 100 spectra) between that quantity and the radiance measured at $2600 \mathrm{~cm}^{-1}$, corrected to remove dependence on surface temperature. The correlation plot used is shown in Figure 1. Second, the other variables of the state vector (surface temperature and $\mathrm{CO}_{2}$ column) are readjusted slightly using the spectral range from 2040 to $2230 \mathrm{~cm}^{-1}$ including the 1-0 lines of CO. 


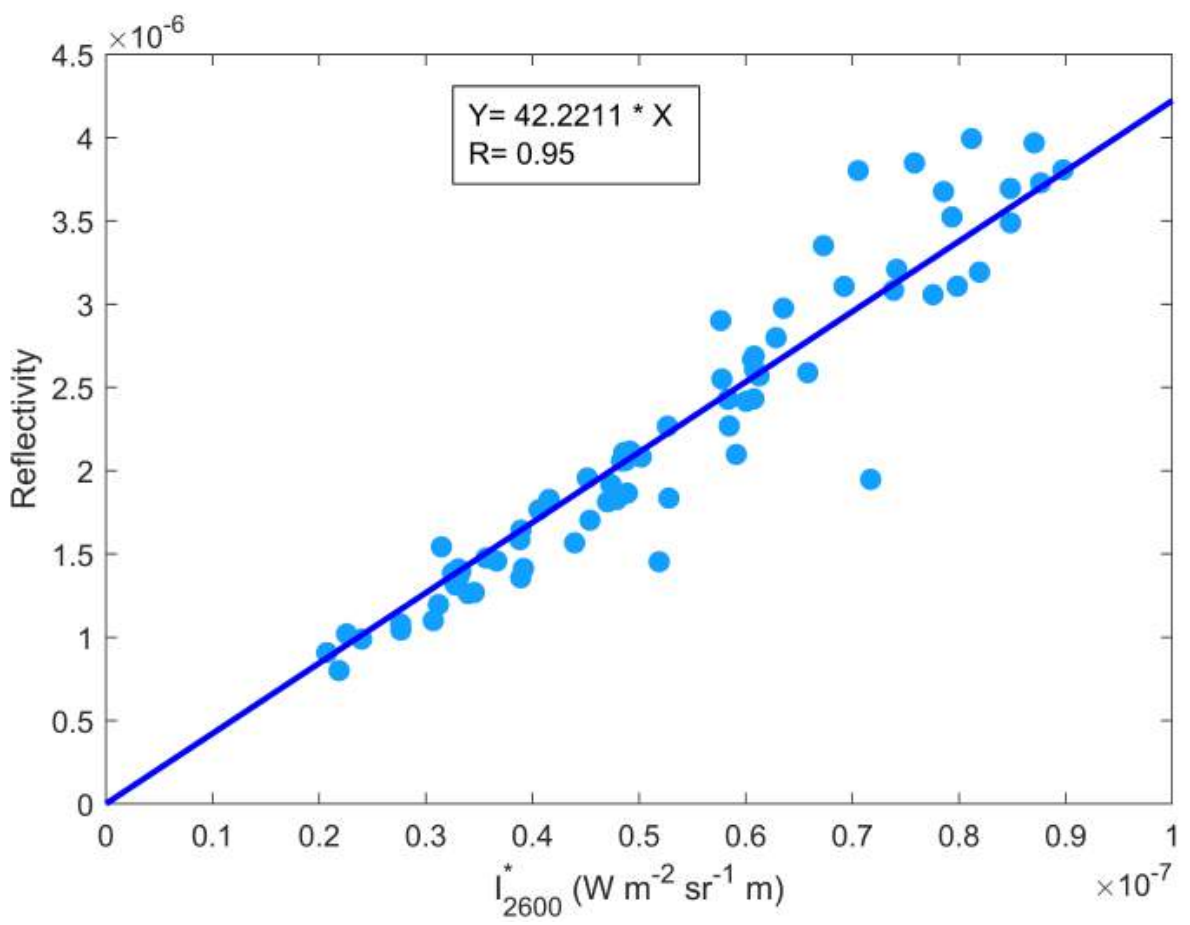

Figure 1. Regression plot between the effective reflectivity and radiance at $2600 \mathrm{~cm}^{-1}$, (corrected for the blackbody radiance at the temperature of the surface $\left.\mathrm{T}_{\mathrm{s}}\right) ; I_{2600}^{*}=I_{2600}-\mathrm{B}_{2600}\left(\mathrm{~T}_{\mathrm{s}}\right)$ and $\mathrm{R}$ is the correlation coefficient.

These changes to the retrieval methodology allow to reduce the computational time by almost one order of magnitude with only marginal differences on the CO column and no bias.

A post-filtering was applied to remove spurious results. Specifically the results were rejected when a degree of freedom for signal (DOFS) for CO was lower than 0.6, when a negative reflectivity or radiance at $2600 \mathrm{~cm}^{-1}$ was found (very weak signal) and when the root-mean squares (RMS) of the residual was higher than $0.058 \mathrm{erg} \mathrm{s}^{-1} \mathrm{sr}^{-1} \mathrm{~cm}^{-2} \mathrm{~cm}$ (equivalent to 2 times the measurement noise applied in the OEM). Around 102.000 spectra are kept after post-filtering. Figure 2 illustrates the quality of the fit for one example among selected spectra, with indications for branches of the $\mathrm{CO}$ band and for $\mathrm{CO}_{2}$ lines. 

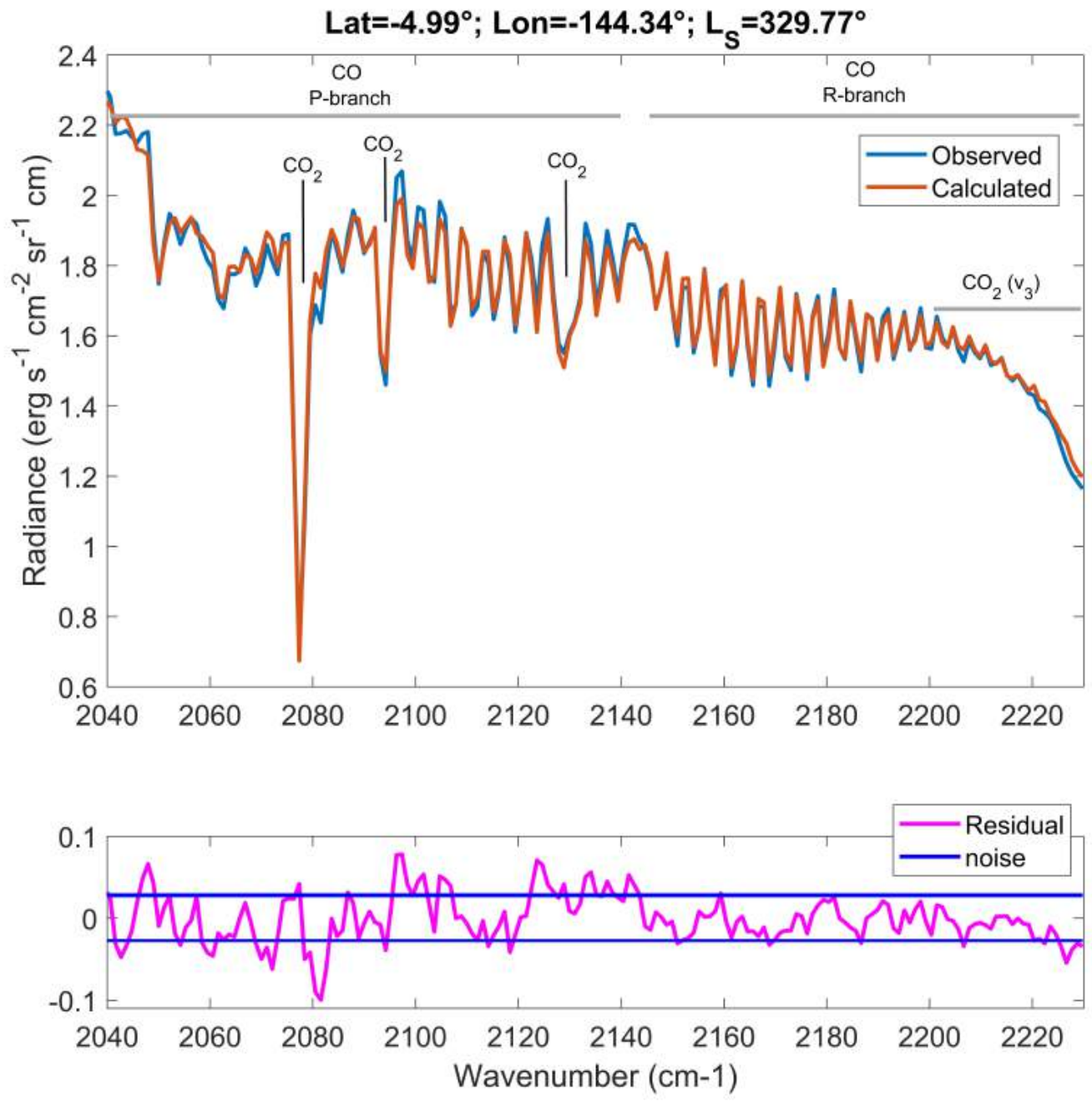

Figure 2. Example fit among the spectra analysed.

\section{Results and discussion}

The results, which use 7 years of the CO PFS measurements together, are shown first in terms of seasonality, per latitude bands, then in terms of spatial variability. The variability of column-averaged VMRs of these results are compared with M. D. Smith et al. (2018), the most recent global analysis of CO on Mars. A comparison with the GEM model is also provided; details on the model and its specific set up for CO can be found in Daerden et al. (2019). Briefly, in the GEM GCM, CO is treated as a non-condensable tracer undergoing photochemical processes, but effectively it is a long-lived tracer, consistent with its estimated lifetime of $\sim 5$ terrestrial years (Lefèvre and Krasnopolsky (2017). Atmospheric photochemistry. In: Haberle et al. (2017) (Eds.), The Atmosphere and Climate of Mars, Cambridge University Press, pp. 405-432.).

$\mathrm{CO}_{2}$ is the main constituent of the Martian atmosphere, yet it follows a strong annual cycle of deposition and sublimation with up to $30 \%$ of mass exchange between the atmosphere and the surface. Enrichment of air with non-condensable gases occurs when $\mathrm{CO}_{2}$ is removed from the atmosphere upon deposition (and vice versa for gas depletion upon $\mathrm{CO}_{2}$ ice sublimation). The volume mixing ratios (VMR) of the other species that do not condense 
with $\mathrm{CO}_{2}$ are then updated proportionally in order with the removed $\mathrm{CO}_{2}$ molecules. This leads to a strong enrichment in the polar winter, as was seen in polar argon observations (Sprague et al., 2004). When the polar winter vortex breaks up, the enriched air starts to leak to lower latitudes. This feature was predicted by models, and first seen in CRISM CO observations (M. D. Smith et al., 2009, 2018).

GCMs have a coarse grid (typically a few degrees in latitude) and cannot maintain such strong meridional gradients, as air will be readily mixed over the area of a model grid cell. This was well demonstrated in model studies of the polar vortex by Seviour et al. (2017). Their Fig. A1 shows the numerical leaking of the vortex for different model resolutions. Their lowest resolution is still finer than our resolution. Problems related to the meridional mixing of enriched air were also discussed in e.g. Nelli et al. (2007) and Lian et al. (2012). GCMs use approximations in their parameterizations for gas enrichment to deal with these problems. There are currently three parameterizations published: Forget et al. (2008), Lian et al. (2012), and the one in GEM-Mars (Neary \& Daerden, 2018; M. D. Smith et al., 2018; Daerden et al., 2019). The implications of such parameterizations is that the enrichment in the polar regions is smaller in amplitude than in reality: the enhancement factor is typically $\sim 3$ (see cited papers), while Lian et al. (2012) could reach an enrichment factor of $\sim 4$ by an improved advection scheme, compared to $\sim 6$ in argon data (Sprague et al., 2004). This allows the GCMs to provide a simulation of non-condensable tracers which maintains specific features of its seasonal evolution, despite not attaining the high enrichment factors in the polar nights.

The current, simple, GEM-Mars GCM parameterization for non-condensable species was briefly introduced and evaluated in Neary and Daerden (2018); M. D. Smith et al. (2018); Daerden et al. (2019). For completeness, we repeat its main features here. Deposition (sublimation) of $\mathrm{CO}_{2}$ above a model point results in a change of the surface pressure, and so also in a change of the air column density above that point $\left(C_{a i r}\right)$. The dynamical core of the GCM then readjusts the atmospheric mass globally, and the impact of polar $\mathrm{CO}_{2}$ deposition is spread out over the entire planet, as can be seen in the observed surface pressure changes on the global scale. However, a local, polar enrichment or depletion of minor species will not spread out over the entire planet. It will first build up locally and then spread out on a much longer time scale, by normal transport (eddy mixing, global circulation). Because of the definition of the model levels in a GCM in terms of surface pressure (implying that the model levels themselves will be modified upon deposition or sublimation), this local process is difficult to disentangle from the global pressure correction. Forget et al. (2008) and Lian et al. (2012) developed methods using fine-scale mass balances between the old and new model levels to treat this problem in their GCMs. In GEM-Mars we apply a simple parameterization that corrects the vmr after deposition (or sublimation) in two steps. In a first step, the VMR of $\mathrm{CO}_{2}\left(\chi_{\mathrm{CO}_{2}}\right)$ is modified uniformly over all model levels such that the change in total column is entirely attributed to $\mathrm{CO}_{2}: \chi_{\mathrm{CO}_{2}}^{\prime}=\chi_{\mathrm{CO}_{2}} \times \frac{\mathrm{C}_{\mathrm{air}}-\mathrm{C}_{\mathrm{non}-\mathrm{CO}_{2}}}{\mathrm{C}_{\mathrm{CO}_{2}}}$, with $\chi_{\mathrm{CO}_{2}}^{\prime}$ the updated VMR of $\mathrm{CO}_{2}$, and $C_{q}$ the column-integrated density of quantity q. The VMR of the other species is updated to ensure that the sum of all VMR equals unity. Such a uniform VMR correction follows the seasonal variation of the pressure, but it will not lead to strong enrichment and it does not take into account the vertical structure of $\mathrm{CO}_{2}$ deposition. To further refine the simulation, an additional, altitude-dependent correction is applied, in which the VMR of $\mathrm{CO}_{2}$ is modified at those vertical model levels $\mathrm{k}$ where actual $\mathrm{CO}_{2}$ ice deposition occurred: $\chi_{C O_{2}, k}^{\prime}=\chi_{C O_{2}, k} \times\left(1-\alpha_{d} \times \frac{\Delta n_{d, i c e, k}}{n_{d, a i r, k}}\right)$, with $\Delta n_{d, i c e, k}$ the number density of $\mathrm{CO}_{2}$ gas that deposits into ice in the grid cell at level $\mathrm{k}$, and $n_{d, a i r, k}$ the air number density. The VMR of the other species are updated again to ensure that the sum of all VMR equals unity. This second step makes the VMR correction also dependent on the altitudes where deposition occurs. The factor $\alpha_{d}$ is a parameter that allows to set the strength of the enrichment, and so to control the time scale of the polar enrichment over the winter, and of the leaking of enriched air to lower latitudes, in comparison with data. In the case that $\mathrm{CO}_{2}$ sublimates from the surface, rather than assuming all the 
released $\mathrm{CO}_{2}$ would go into the shallow lowest model level $(1.5 \mathrm{~m}$ thick), we assume it is readily mixed over the lowest 40 model levels (up to $\sim 27 \mathrm{~km}$ ) with a similar expression: $\chi_{\mathrm{CO}_{2}}^{\prime}=\chi_{\mathrm{CO}_{2}} \times\left(1-\alpha_{s} \times \frac{\Delta C_{i c e}}{C_{a i r}}\right)$, with $\Delta C_{i c e}$ the change in total column density of $\mathrm{CO}_{2}$ ice particles (negative if ice sublimates), $C_{\text {air }}$ the air column density, and $\alpha_{s}$ another parameter. The parameters $\alpha_{d}$ and $\alpha_{s}$ do not necessarily have the same values, as they describe different processes that involve different time scales, but they should be well balanced to ensure that the total atmospheric content of noncondensing species does not change. While these parameters introduce an extra degree of uncertainty in the model, they allow to provide a currently working simulation, while a more advanced treatment can be developed in the future.

A first resulting simulation using this parameterization was presented in M. D. Smith et al. (2018); Daerden et al. (2019). This reproduced some general features of the observed CO cycle. Because CRISM can not observe in the polar night, comparisons were not possible there, but the model simulated the depletion during polar spring and summer, and the leaking of enriched air to lower latitudes, where it causes a maximum in the middle of the Martian year that is stronger than solely based on the surface pressure change alone. Later it was found than an improved model-data comparison was obtained by lowering the initial CO abundances overall by $10 \%$ (followed by a multi-annual spin-up to allow the atmosphere to adapt itself to these new conditions). The simulation was also improved when the parameters of the non-condensable gas enrichment were modified from $\alpha_{d}=$ $0.025, \alpha_{s}=0.030$ (M. D. Smith et al., 2018) to $\alpha_{d}=0.032, \alpha_{s}=0.043$. It is this slightly improved simulation that is presented in this paper. Remaining model-data differences that show up in the detailed comparison (see below) will be useful to improve the model and its parameterization in the future. E.g., the parameterization is sensitive to the accurate simulation of $\mathrm{CO}_{2}$ polar cap deposition/sublimation in the model. While in general the simulation of the polar $\mathrm{CO}_{2}$ ice mass looks good (see Neary and Daerden (2018), their Fig. 15, to be compared to data in Kelly et al. (2006), their Fig. 4a, or Haberle et al. (2008), their Fig. 3), it seems from the comparisons to CO data below that small imperfections can produce a visible bias for $\mathrm{CO}$. The implementation and evaluation of potential model improvements in order to further minimize such model-data biases, however, is beyond the scope of this paper.

\subsection{Climatology of retrieved CO}

Figure 3 shows the latitudinal distribution of the retrieved $\mathrm{CO}$ averaged over the 7 years of PFS observations, both in terms of total columns (top, left) and column-averaged VMRs (top, right), as a function of solar longitude $L_{S}$. The averaged VMR has been calculated by dividing the total column of $\mathrm{CO}$ by that of $\mathrm{CO}_{2}$ retrieved simultaneously. Figure $3 \mathrm{c}$ shows, for each cell, the average of the total errors (\%) associated to the individual retrieved CO columns falling in the cell. The number of measurements per cell (bottom, right) is shown in Figure 3d. The CO columns, VMRs, errors and number of measurements have been averaged in cells of $5^{\circ}$ latitude and $5^{\circ} L_{S}$. It is important to stress that the total uncertainty on individual retrieved $\mathrm{CO}$ columns includes the retrieval uncertainty related to the Optimal Estimation method, which accounts for the measurement error and the smoothing error (see Bouche et al. (2019) for details). Two other sources of uncertainties have been taken into account in the total error on the CO column: 1) the uncertainty on the considered temperature profile (6\% on average, as estimated in S. Bauduin et al., personal communication), and 2) the uncertainty due to the use of an average representation for the instrumental line shape of PFS (ILS) instead of an observation-dependent ILS as recommended by the PI team (estimated at around $13 \%$; not shown). Overall the total errors on individual CO columns are on average around 20-25\%. It is worth to stress that Figure $3 \mathrm{c}$ does not show the error on the average CO column, which is expected to be lower given the number of spectra averaged together (the random part of the total uncertainty decreases as the square root of the number of spectra averaged together, Figure 3d). 


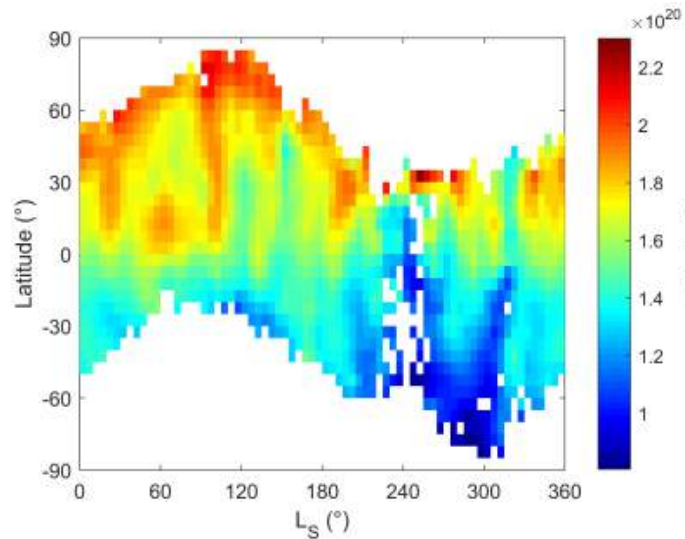

(a)

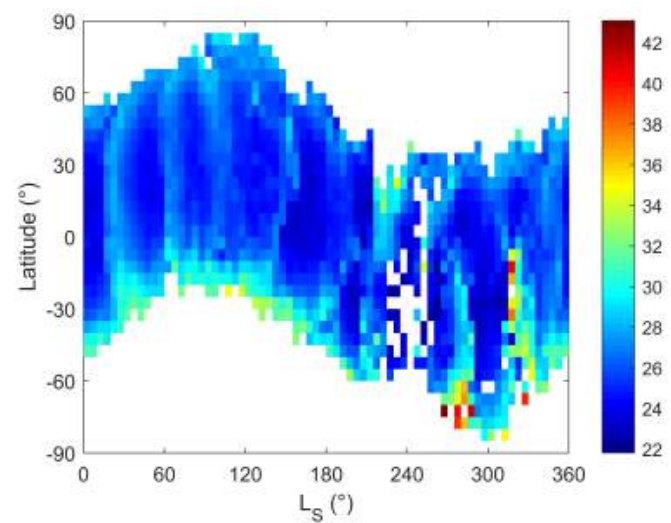

(c)

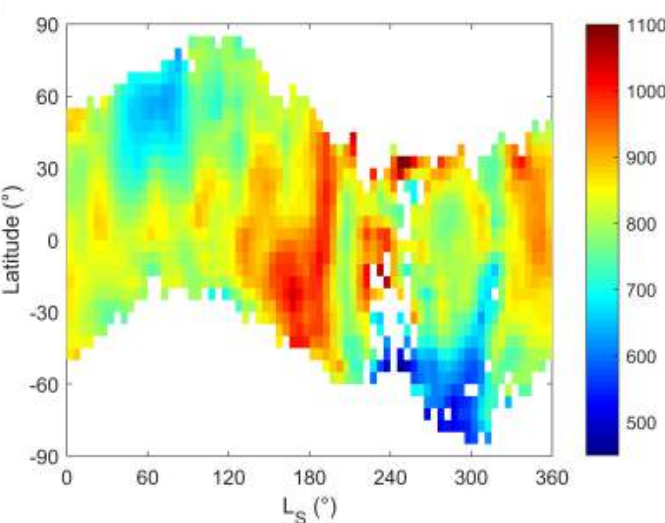

(b)

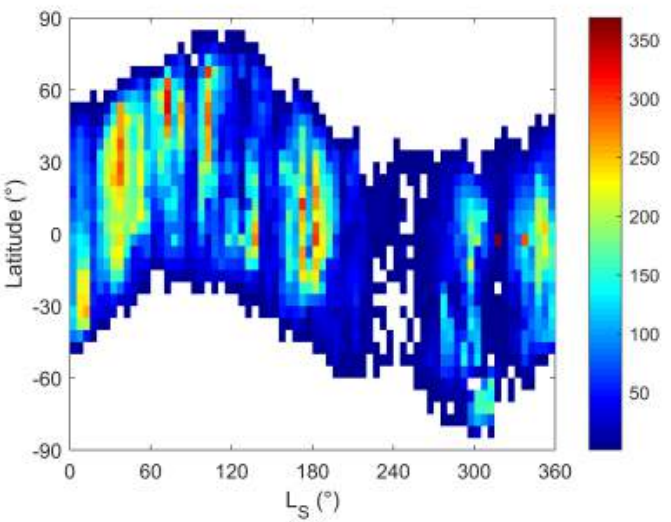

(d)

Figure 3. Total columns in molecules $\mathrm{cm}^{-2}$ (panel a) and mean column-VMR in ppm (panel b) averaged in $5^{\circ}$ latitude and $5^{\circ} L_{s}$ cells, taking into account all successful retrievals from the 7 years of PFS measurements. Panel d gives the number of measurements per cell, and panel c shows the average total uncertainties (\%) for each grid cell. They are calculated as the average of the total uncertainties estimated for each individual PFS observations falling in a cell, and hence correspond to the average uncertainty on a single measurement. This total uncertainty involves the retrieval error, which includes the smoothing and measurement errors (see Bouche et al. (2019) for details), the error due to the uncertainty on the temperature profile and the error due to the formulation of the ILS (see text for details). Note that the distribution (panels a and b) are smoothed to better reveal the main patterns of seasonality. The smoothing used is a fully automated and robust procedure for uniformly sampled datasets, and based on penalized least squares method. It is done by means of the discrete cosine transform. The smoothing parameter used in the algorithm for this work is fixed to 0.3 , this corresponds to a weakly smoothed output. A full description of the algorithm in the smoothing function can be found in Garcia (2010) and Garcia (2011).

It is important to note that the number of measurements (shown in Figure 3d) per grid cell is highly variable, between 2 to several hundreds. The main reason for the small number of measurements, especially close to the winter polar regions, is the weakness of the PFS radiance during the colder periods and in the absence of solar light, which is such that CO lines hardly emerge from the noise and that the resulting spectra are rejected using the selection criteria used here. In order to better highlight the main patterns of 
seasonality with these variable number of measurements per grid cell, a slight smoothing of the columns and the column-averaged VMRs has been applied in Figure 3a and Figure 3b. For long periods of the years and latitudes between $30^{\circ}$ and $90^{\circ}$ of the hemisphere in winter, the situation is often such that no retrievals at all have been performed (white areas in Figure 3); for instance there are only a handful of successful retrievals above $80^{\circ}$ latitude, which correspond to polar springs/summers. Another reason for the lack of $\mathrm{CO}$ retrievals between $30^{\circ} \mathrm{S}$ and $30^{\circ} \mathrm{N}$ for $200-250^{\circ} L_{S}$ is the dusty season. Indeed, a lot of PFS spectra have been rejected because they were associated to large $\tau_{\mathrm{d}}$. While the different selection criteria are justified from a retrieval point-of-view, they are likely to bias the cell-averaged $\mathrm{CO}$ columns. On the one hand, the selection based on the CO line strength rejects spectra associated with potentially low CO abundance, leading to an overestimation of the CO cellaverage. On the other hand, the rejection of spectra due to the criterion on $\tau_{\mathrm{d}}$ might result in non-representative $\mathrm{CO}$ average abundances for some cells when the number of observations kept is too small. This has to be kept in mind in the comparison to other dataset, especially model-based which do not suffer from the same bias.

In terms of total columns, we find that they vary between $8 \times 10^{19}$ and $2.3 \times 10^{20}$ molecules $\mathrm{cm}^{-2}$, with a global average value of $1.6 \times 10^{20}$ molecules $\mathrm{cm}^{-2}$. There is a clear influence of orography on the distribution, which will be discussed in Section 3.2. The column-averaged VMRs remove this dependence and the distribution is easier to discuss in terms of seasonality. On a global average, we calculate a mean VMR of around $820 \mathrm{ppm}$, which is in the range of those usually reported from ground or space-based instruments (Krasnopolsky, 2003; Billebaud et al., 2009; Sindoni et al., 2011; M. D. Smith et al., 2009, 2018). We note, however, a peculiar feature at $\mathrm{L}_{S}=200-210^{\circ}$ corresponding to a sharp drop of the CO VMR. At the moment, the reason for this drop is not very clear. A combination of different factors could explain this likely unrealistic feature. For instance, as explained above, some of the averaged CO VMR could be not representative of the real CO column because of a too low number of observations averaged together. This is probably the case here given the small number of retrieved CO column averaged together for that region (Figure 3d). It also seems that part of the fits in this region are of somewhat less quality than for other region. This feature has therefore to be taken with caution in the interpretation of the results and in comparison with other datasets or models. To discuss the seasonality, the CO columnaveraged VMR is plotted against $\mathrm{L}_{S}$ for 9 latitude bands of $20^{\circ}$ in Figure 4, similarly to what has been done in M. D. Smith et al. (2018) with CRISM observations (Figure 11 from M. D. Smith et al. (2018)). The discussion is divided according to the latitude bands in the following subsections. 

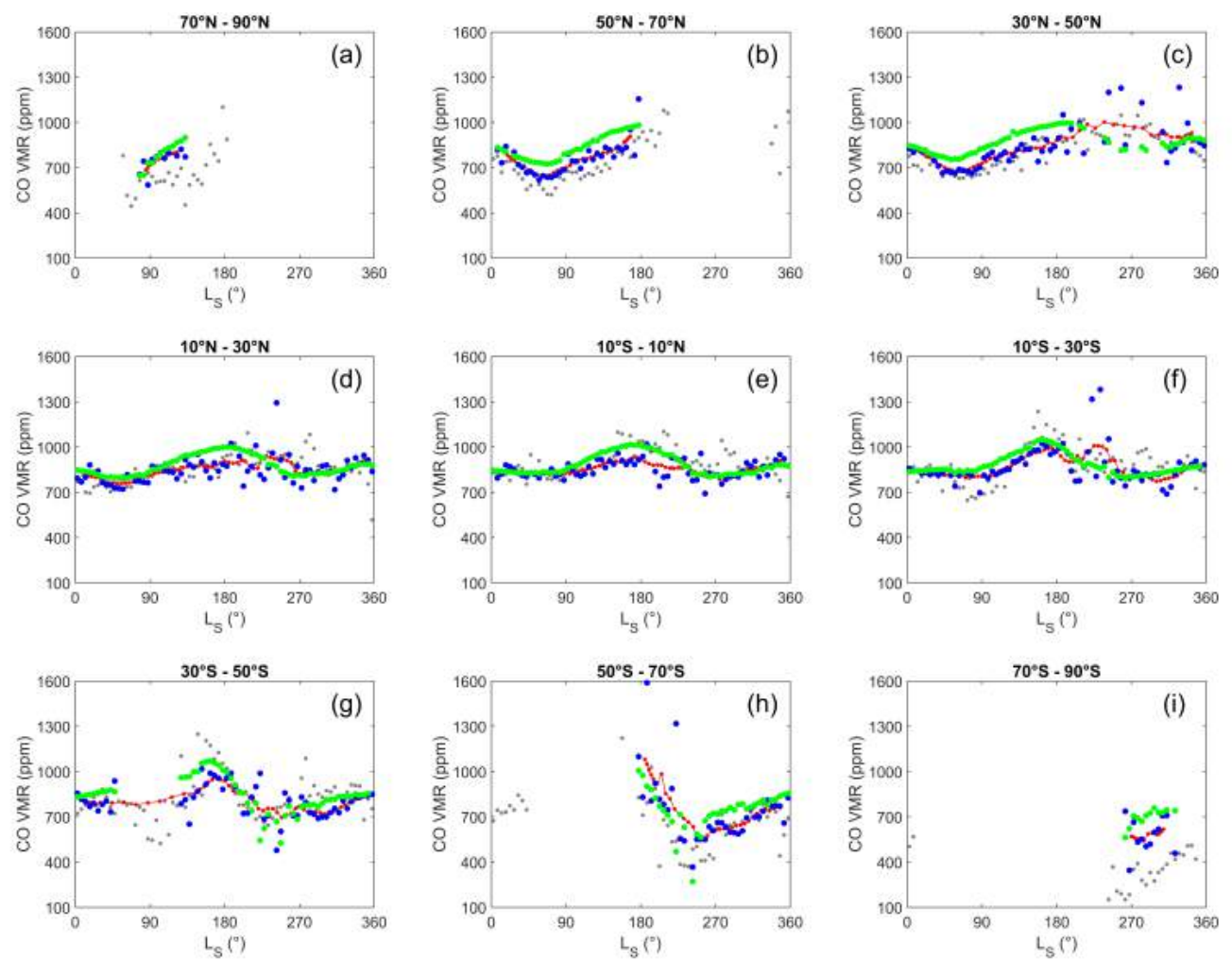

Figure 4. Column-averaged CO VMRs retrieved (in blue) from PFS as a function of $\mathrm{L}_{S}$ for 9 latitude bands. They are calculated for boxes of $5^{\circ} \mathrm{L}_{S}$. The red line is a running-average, which computes a centered moving average by sliding a window of $35^{\circ} \mathrm{L}_{S}$ along the data points. The green points are the column-averaged CO VMRs simulated by the GEM-Mars GCM model updated from Neary and Daerden (2018) as explained in the text from Daerden et al. (2019) and grey points are the column-averaged CO VMRs retrieved from CRISM by M. D. Smith et al. (2018).

\subsubsection{Polar and subpolar latitudes bands}

At subpolar latitudes $\left(50^{\circ}-70^{\circ}\right.$ bands, Figure $4 \mathrm{~b}$ and Figure $\left.4 \mathrm{~h}\right)$ in spring/summer, the retrieved VMRs (blue dots in Figure 4) are significantly lower than the mean VMR (820 ppm), due to the sublimation of $\mathrm{CO}_{2}$ ice. The minimum retrieved values reach almost $620 \mathrm{ppm}$ in the Northern hemisphere (Figure 4b) and less in the Southern hemisphere (Figure 4h), around $370 \mathrm{ppm}$. As already observed in other studies, this North/South difference is due to the difference in the $\mathrm{CO}_{2}$ ice mass accumulating at the poles at the cold season (Krasnopolsky, 2003). Also, summer solstice in the Southern hemisphere occurs during perihelion, when the distance between Mars and the Sun is the shortest. The stronger solar radiation during Southern summer can also explain the more severe depletion of CO at the summer South pole compared to the North pole at the same season. Note that we observe these very low $\mathrm{CO}$ values before the summer solstice in both hemispheres, but slightly earlier for the Northern hemisphere $\left(\right.$ at $\mathrm{L}_{S} \sim 60^{\circ}$ ) than for the Southern hemisphere (at $\mathrm{L}_{S} \sim 250^{\circ}$ ). According to Figure $3 \mathrm{~b}$, the decrease of the CO VMR for the Northern hemisphere seems to be also observed at mid-latitudes (down to $30^{\circ} \mathrm{N}$ ), while the very low CO VMRs seem confined to latitudes below $45^{\circ}-50^{\circ} \mathrm{S}$ in the Southern hemisphere. Regarding the band $70^{\circ}-90^{\circ}$, only a few retrieved CO VMRs are available after the summer solstice for both hemisphere, due to the reasons already exposed above. For the period covered, 
the largest VMRs are around $840 \mathrm{ppm}$ and $710 \mathrm{ppm}$ for the North and the South poles respectively (Figure 4a and Figure 4i respectively).

Comparing to CRISM CO measurements from M. D. Smith et al. (2018) for the same latitude bands, we find a general agreement for the $50^{\circ} \mathrm{N}-70^{\circ} \mathrm{N}$ band, in terms of absolute values (average difference around $80 \mathrm{ppm}$ ) and amplitude of the seasonal cycle. At polar latitudes $\left(70^{\circ}-90^{\circ}\right)$, our retrieved values are typically larger by 100 to $200 \mathrm{ppm}$ in the Northern hemisphere (this is almost within the error of CRISM where the estimated uncertainty in the binned retrievals is $100 \mathrm{ppm}$ ) and to $300 \mathrm{ppm}$ in the Southern hemisphere. More especially, values as low as $200 \mathrm{ppm}$ are retrieved by CRISM in the Southern hemisphere but not in our work. This difference might be explained by our selection, privileging high $\mathrm{CO}$ abundance and rejecting low $\mathrm{CO}$ signals, thus resulting probably in overestimated $\mathrm{CO}$ averages for some periods and regions. In addition, in this region there are less PFS measurements and the retrieval errors are larger (see Figure 3c). We also retrieve larger VMRs than CRISM for the $50^{\circ}-70^{\circ}$ band in the Southern hemisphere close to the summer solstice (around $550 \mathrm{ppm}$ for this work between $225^{\circ}-270^{\circ} \mathrm{L}_{S}$ against $400 \mathrm{ppm}$ for CRISM), which could be due in part to the high-bias just mentioned.

Compared to the GEM-Mars model (green dots in Figure 4) for spatially geolocated grid-cells, our retrievals show a better match in the $70^{\circ} \mathrm{N}-90^{\circ} \mathrm{N}$ and at the beginning of the Northern spring for the $50^{\circ}-70^{\circ}$ band. The difference between our retrieved values and model values is typically within $10-150 \mathrm{ppm}$. The seasonal variability (timing and amplitude) for the $50^{\circ}-70^{\circ}$ band of both hemisphere is in general agreement with the model, except that, for the Northern hemisphere, the amplitude of the CO VMR decrease at the beginning of the year is larger for the retrieved values (from 800 down to $600 \mathrm{ppm}$ ) than for the modelled values. We attribute this low amplitude in the decrease in the model to an imperfect simulation of the Northern springtime deposition/sublimation of the $\mathrm{CO}_{2}$ seasonal cap. Possible reasons for this are the simplified definition of the permanent cap area, the implementation of thermal inertia and surface fields, or the prescription of subsurface ice (Neary \& Daerden, 2018; M. D. Smith et al., 2018). This case is one example where the comparison of $\mathrm{CO}$ to the new dataset can help to improve the model in the future.

\subsubsection{Mid-latitudes bands}

At mid-latitudes $\left(30^{\circ}-50^{\circ}\right.$ bands, Figure $4 \mathrm{c}$ and Figure $\left.4 \mathrm{~g}\right)$, we observe as well a clear seasonality in both hemispheres. In the Northern hemisphere, it is characterized by a marked minimum between $\mathrm{L}_{S}=50^{\circ}-70^{\circ}$ (local spring) with VMRs around $660 \mathrm{ppm}$, and larger but very scattered values around $1000 \mathrm{ppm}$ in autumn and winter (several values reaching more than $1100 \mathrm{ppm})$. In the Southern hemisphere, there is a maximum at similar values (1000 ppm) during a relatively short period in the local winter $\left(150^{\circ}-180^{\circ} \mathrm{L}_{S}\right)$. It is followed by a rapid decrease to values around $700 \mathrm{ppm}$ (moving average) from $\mathrm{L}_{S} \sim 240^{\circ}$ to $\mathrm{L}_{S} \sim 300^{\circ}$. There is a second maximum at the end of the Martian year, but with values not higher than $850 \mathrm{ppm}$.

This behaviour is very similar to that reported by M. D. Smith et al. (2018) using CRISM for both hemispheres. Especially, the sharp peak of high CO in local winter in the Southern hemisphere is similarly captured. However, we cannot confirm the lowest values found by CRISM in local late autumn/beginning winter due to a lack of measurements in our case. In comparison with the GEM-Mars model, we find a general agreement for both hemispheres in terms of the amplitude of the seasonal cycle, except for the range of $\mathrm{L}_{S}$ where too few and/or scattered values are retrieved $\left(\mathrm{L}_{S}=210^{\circ}-345^{\circ}\right.$ in the Northern hemisphere; $\mathrm{L}_{S}=50^{\circ}-120^{\circ}$ and $\mathrm{L}_{S}=200^{\circ}-280^{\circ}$ in the Southern hemisphere). Between $200^{\circ}$ and $300^{\circ}$ of $\mathrm{L}_{S}$, despite the limited set of retrieved VMRs, it is worth pointing that the latter are frequently higher than in the model with differences that can go up to $\sim 400 \mathrm{ppm}$. For the rest of the $\mathrm{L}_{S}$ range, where the seasonal cycle is better matching, the model is larger by 10 to $150 \mathrm{ppm}$ compared to the PFS-derived values. In the North, this difference 
is a result of the difference at higher latitudes which is progressing towards lower latitudes. In the South, the difference is comparable to the differences between the PFS and CRISM datasets, making it more difficult to draw conclusions at this stage.

\subsubsection{Equatorial latitude bands}

Between $30^{\circ} \mathrm{N}$ and $30^{\circ} \mathrm{S}$, the retrieved CO column-averaged VMRs seem less prone to seasonal variations, with changes of generally less than $100 \mathrm{ppm}$ around a mean value of 900 ppm (Figures $4 \mathrm{~d}-\mathrm{f}$ ). However, in the $10^{\circ} \mathrm{S}-30^{\circ} \mathrm{S}$ band, we distinctly observe a period of higher CO VMRs that starts at $\mathrm{L}_{S} \sim 100^{\circ}$ with values around $800 \mathrm{ppm}$ to reach a maximum of 1000 ppm at $\mathrm{L}_{S} \sim 160^{\circ}$. This is also evident in Figure $3 \mathrm{~b}$. The high CO VMRs for the $10^{\circ} \mathrm{S}-30^{\circ} \mathrm{S}$ band is well observed between $120^{\circ}-190^{\circ} \mathrm{L}_{S}$. The band $10^{\circ} \mathrm{S}-10^{\circ} \mathrm{N}$ present also higher CO values for the same $\mathrm{L}_{S}$ range but to a lesser extent, explaining the smoother increase of CO VMRs for that band (Figure 4e). Finally, in Figure 3b, we can see that the high CO values cross the equator at around $\mathrm{L}_{S}=170^{\circ}$ and extend to $40^{\circ} \mathrm{N}$. This explains the higher $\mathrm{CO}$ VMRs retrieved for the $10^{\circ} \mathrm{N}-30^{\circ} \mathrm{N}$ band for $\mathrm{L}_{S}=170^{\circ}-250^{\circ}$ (Figure $4 \mathrm{~d}$ ). These elevated CO VMRs in the equatorial region are possibly due to the transport of CO-enriched air from the Southern polar winter region (condensation of $\mathrm{CO}_{2}$ ) to the North. Note finally that, in Figure $3 \mathrm{~b}$, a region of high CO VMRs is observed between $30^{\circ} \mathrm{S}$ and $10^{\circ} \mathrm{N}$ for $\mathrm{L}_{S}=200^{\circ}-250^{\circ}$ (also well observed in Figure 4f). These have never been reported in other studies and were unexpected. It is very likely that the CO averaged VMRs are not representative and bias are high for this area due to the very low number of PFS observations analysed for this region (Figure 3d).

The comparison of our retrieved CO VMRs with those measured from CRISM (M. D. Smith et al., 2018) is again favorable (mean difference of $20 \mathrm{ppm}$ and standard deviation between the two datasets lower than $115 \mathrm{ppm}$ ). The timing of the different increases of CO VMRs is nicely matching between the two datasets: For instance, the two instruments observe the highest CO VMRs for the $10^{\circ} \mathrm{N}-30^{\circ} \mathrm{N}$ band later (after the local autumn equinox) than for the $10^{\circ} \mathrm{S}-10^{\circ} \mathrm{N}$ and $10^{\circ} \mathrm{S}-30^{\circ} \mathrm{S}$ bands (before the local autumn equinox). The amplitude of the CO increase inferred from CRISM observations is however larger than what we measure. The difference is especially marked for the $10^{\circ} \mathrm{N}-30^{\circ} \mathrm{N}$ and $10^{\circ} \mathrm{S}-10^{\circ} \mathrm{N}$ bands where the amplitudes differ by about $100 \mathrm{ppm}$ (900 ppm for our moving average and $1000 \mathrm{ppm}$ for CRISM). However, this difference remains below the combined uncertainty of both datasets. A better match is found for the $10^{\circ} \mathrm{S}-30^{\circ} \mathrm{S}$ band with maxima CO VMRs around $1000 \mathrm{ppm}$.

In comparison with the GEM model, for the 3 equatorial bands, we find a good correspondence for the beginning and the end of the Martian year, i.e. for $\mathrm{L}_{S}=0^{\circ}-80^{\circ}$ and for $\mathrm{L}_{S}=270^{\circ}-360^{\circ}$. Larger differences are observed in the middle of the year for the $10^{\circ} \mathrm{N}-30^{\circ} \mathrm{N}$ and $10^{\circ} \mathrm{S}-10^{\circ} \mathrm{N}$ bands, especially regarding the amplitude of the seasonal cycle. Differences of around $100 \mathrm{ppm}$ are observed for the largest VMRs of CO (1000 ppm for GEM-Mars and $900 \mathrm{ppm}$ for the moving average). Note that the $\mathrm{L}_{S}$ range where these larger differences are observed $\left(\mathrm{L}_{S}=120^{\circ}-270^{\circ}\right)$ is a range where the retrieved VMRs show a large scatter. For the $10^{\circ} \mathrm{S}-30^{\circ} \mathrm{S}$ band, the seasonal cycle is very similar in terms of amplitude, except for $\mathrm{L}_{S}=200^{\circ}-250^{\circ}$ where the retrieved VMRs show larger values, possibly due to the reason discussed above (averages not representative). Regarding the timing, the model simulates the increase in $\mathrm{CO}$ earlier than is measured (from $\mathrm{L}_{S} \sim 80^{\circ}$ compared to an increase at $\mathrm{L}_{S}$ $\sim 100^{\circ}$ for PFS). As explained in the introduction of Section 3, the low latitude increase of $\mathrm{CO}$ is a direct consequence of the polar enrichment, the break-up of the winter vortex, and the leaking of enriched air to lower latitudes. The model-data difference seen in this new dataset, could hint at the imperfect simulation of one or each of these processes in the GCM. In any case, the small differences seen here fall within the uncertainties of the parameterization and the applied parameters, and does not allow to draw strong conclusions at this stage. 


\subsection{Spatial variation of retrieved CO}

The maps in Figures 5 and 6 show the spatial distribution of, respectively, the PFSretrieved integrated $0-24 \mathrm{~km}$ column density and CO VMR, separately for each Martian season. The same smoothing function as the one used in Figure 3 has been applied to the retrievals to better show the main spatial patterns.

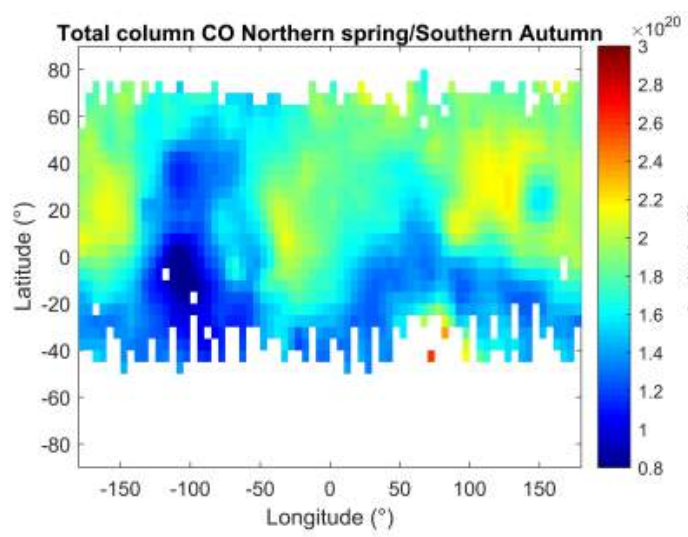

(a)

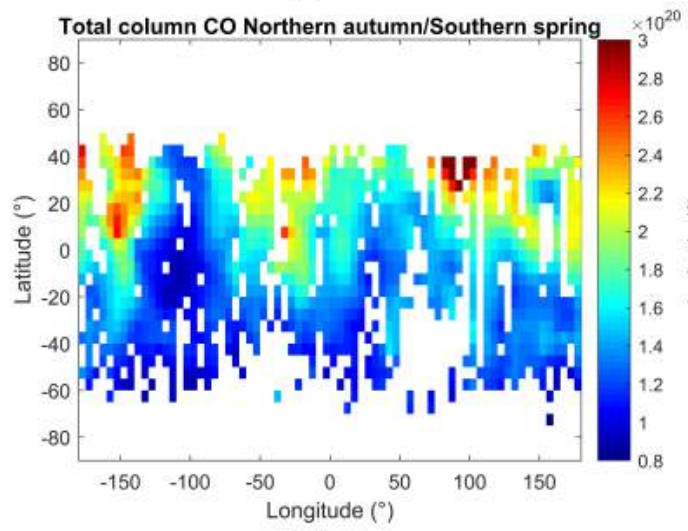

(c)

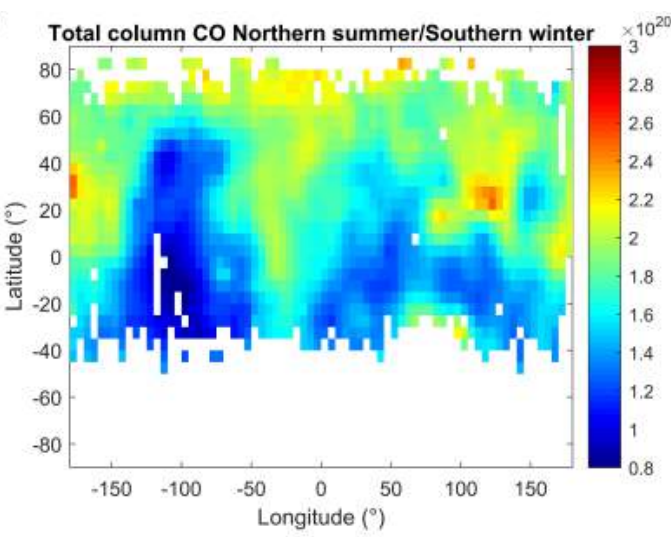

(b)

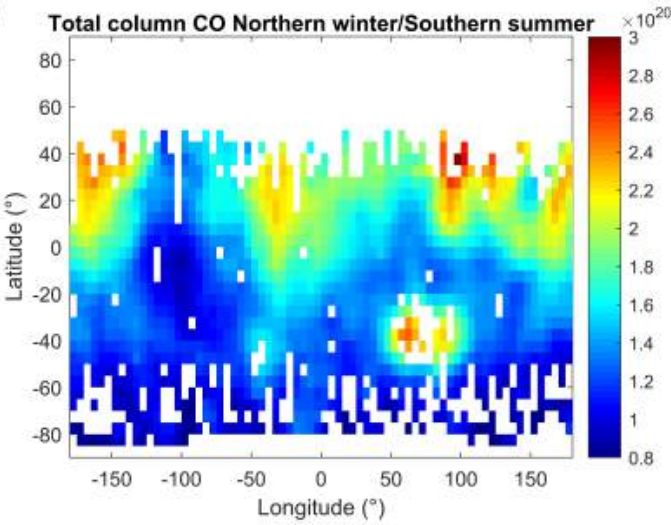

(d)

Figure 5. Distribution of CO column density $\left(\right.$ molecules $\mathrm{cm}^{-2}$ ) of the $0-24 \mathrm{~km}$ retrieved columns from PFS spectra for each season (Northern spring $\mathrm{L}_{S}=\left[0-90^{\circ}\right]$, Northern summer $\mathrm{L}_{S}=\left[90-180^{\circ}\right]$, Northern autumn $\mathrm{L}_{S}=\left[180-270^{\circ}\right]$, Northern winter $\left.\mathrm{L}_{S}=\left[270-360^{\circ}\right]\right)$. The longitudes are East. Note that the distribution are smoothed to better reveal the main patterns of seasonality. The smoothing used is the same as in Figure 3.

The distribution of the CO column density (Figure 5) closely follows the topography of Mars, as recorded by the Mars Orbiter Laser Altimeter (MOLA) (D. E. Smith et al., 2001). This is explained by pressure differences (and so air density differences). Higher column densities are retrieved in high pressure regions, such as in the bottom of the Hellas basin where the columns vary between $1.7 \times 10^{20}$ and $2.5 \times 10^{20}$ molecules $\mathrm{cm}^{-2}$ in Southern summer (other seasons lack measurements). Conversely, the lowest values are associated with mountains and volcanoes like Tharsis, regions where the pressure is lower. The columns in these elevated areas is as low as $8 \times 10^{19}$ molecules $\mathrm{cm}^{-2}$. Hence a dynamic range of column spanning almost one order of magnitude between extremes (from $8 \times 10^{19}$ to $3 \times 10^{20}$ molecules $\mathrm{cm}^{-2}$ ) is retrieved, in comparison to typically a factor 2 to 3 for the VMRs. 


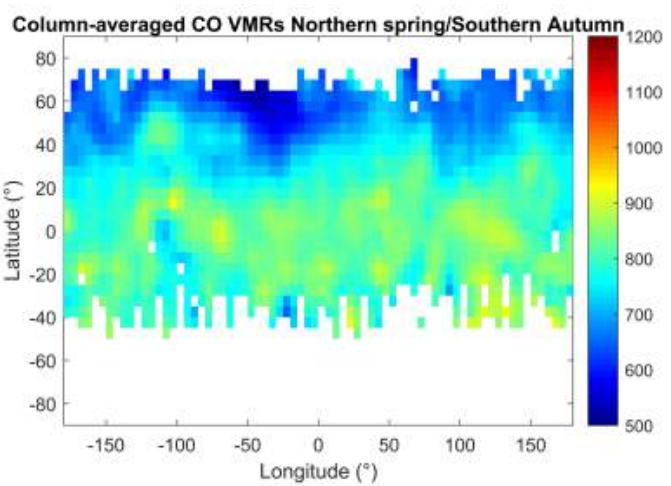

(a)

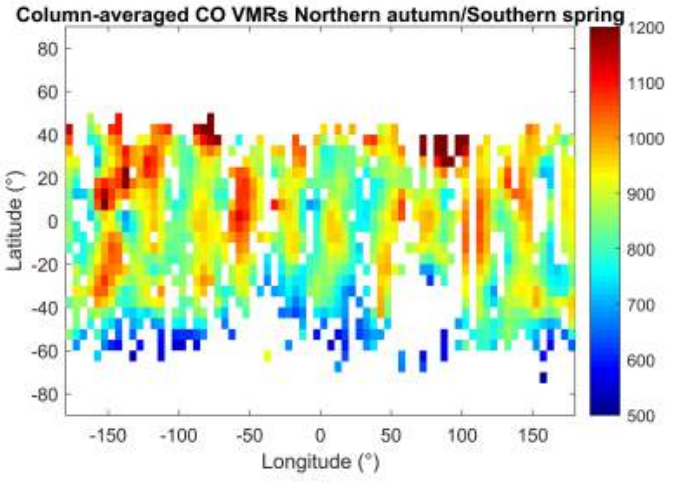

(c)

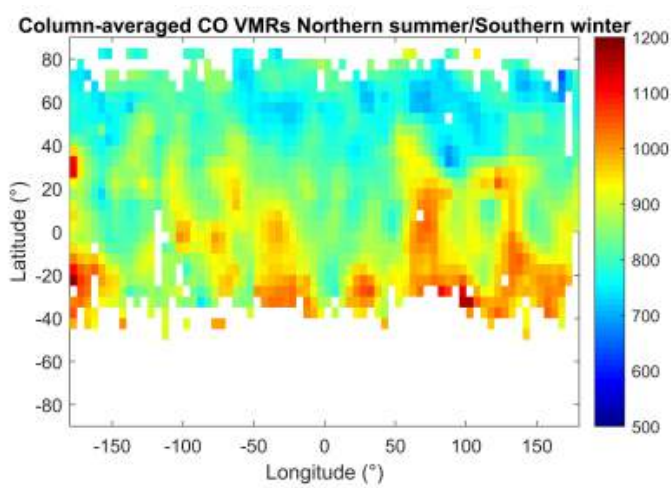

(b)

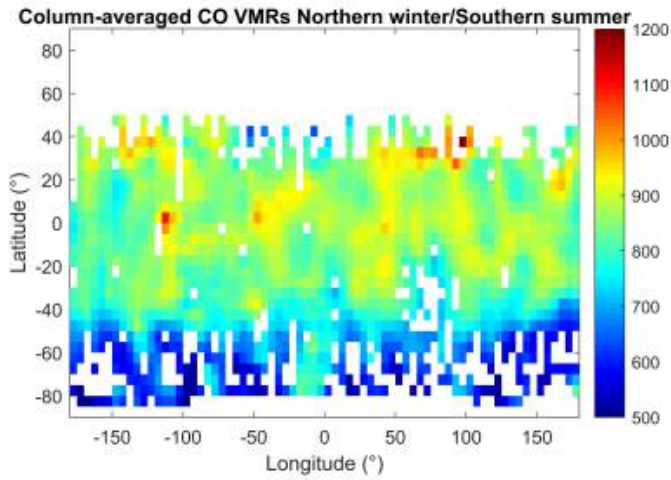

(d)

Figure 6. Distribution of CO column-averaged VMRs (ppm) retrieved from PFS spectra for each season (Northern spring $\mathrm{L}_{S}=\left[0-90^{\circ}\right]$, Northern summer $\mathrm{L}_{S}=\left[90-180^{\circ}\right]$, Northern autumn $\mathrm{L}_{S}=[180$ $\left.270^{\circ}\right]$, Northern winter $\left.\mathrm{L}_{S}=\left[270-360^{\circ}\right]\right)$. The longitudes are East. Note that the distribution are smoothed to better reveal the main patterns of seasonality. The smoothing used is the same as in Figure 3.

In terms of the column-averaged VMRs, the maps in Figure 6 principally reveal the seasonal patterns presented and discussed in section 3.1. The most pronounced ones relate to the condensation/sublimation in polar regions. As pointed out in the previous section, the CO depletion during polar summer is more severe in the Southern hemisphere. Moreover, the depletion of CO lasts two seasons (spring and summer) while it seems to be strong only in spring for the Northern hemisphere. A series of peculiar features emerge from the distributions, some of which may be real while some others could be artifacts related to the selection criteria used and the limited number of successful retrievals in certain areas and periods. To take a couple of examples, at Northern mid-latitudes an enhancement of CO VMR is observed around Alba Patera region $\left(\sim 850 \mathrm{ppm}, \sim 40^{\circ} \mathrm{N} 110^{\circ} \mathrm{W}\right)$ during local spring, while in the Southern hemisphere, one enhancement is observed around Noachis Terra $\left(\sim 800 \mathrm{ppm}, \sim 60^{\circ} \mathrm{S} 10^{\circ} \mathrm{W}\right)$ during local summer. These relatively large values of CO are potentially due to the bias included in our selection criteria where the weak columnaveraged VMRs are excluded in the calculation of mean. Moreover, Alba Patera volcano can have low pressure local circulation during the Northern spring associated with water ice clouds during aphelion.

There are a series of other interesting patterns of spatial variability that are revealed in Figure 6, which occur mainly at latitudes lower than $40^{\circ}$ between $\mathrm{L}_{S}=90^{\circ}$ (beginning of Northern summer/Southern winter) and $\mathrm{L}_{S}=270^{\circ}$ (end of Northern autumn/Southern 
spring). Among these it is worth pointing out the annual maximum values found specifically near to Utopia Planitia region $\left(\sim 35^{\circ} \mathrm{N} 90^{\circ} \mathrm{E}\right)$ in local autumn season with values around $1400 \mathrm{ppm}$. Note that the high CO VMRs values observed in these regions are potentially biased given the low number of retrievals in these cells.

\subsection{Comment on the vertical profiles}

As introduced in Section 2, one particularity of the dataset presented here is that it is obtained from retrieving vertical profiles from individual PFS spectra. Despite the fact that the measurements do not allow to retrieve the $\mathrm{CO}$ abundance at different altitudes independently from one another, we have looked into the profile variability as a function of latitude and season. It is presented in Figure 7. These latitudinal cuts, per $5^{\circ}$ of latitude, show logically the same variability as that of the column-averaged VMRs, with very low values of the VMR at all altitudes in the polar regions in local spring/summer and larger values at mid- and tropical latitudes, especially during Northern summer (Figure 7b) and Northern autumn (Figure 7c). The mid-latitude regions and, in particular, the subtropics, exhibit a strong vertical gradient with large VMRs near the surface $(\sim 1100 \mathrm{ppm})$ decreasing to $700-800 \mathrm{ppm}$ above $6 \mathrm{~km}$, slightly increasing again above. At higher latitudes (especially beyond $50^{\circ}$ ) the profiles seem less variable with height. Even if it has been shown by Daerden et al. (2019) and Holmes et al. (2019) that the CO VMR is probably not uniformly distributed vertically in some regions and in some seasons, these features have to be treated with great care as they may result from the varying vertical sensitivity of PFS rather than by a real variation of the $\mathrm{CO}$ vertical distribution. In order to verify if the retrieved profiles are representative of known processes, we have confronted them to simulated model profiles, for different seasons and latitudes. We recall, however, that we lack data to independently validate the model outputs. The GEM-Mars profiles (Figure 9 and 10 from Daerden et al. (2019)) show a clear latitudinal gradient similar to what we observe but they present a quasi-uniform vertical distribution of CO. We have then made a qualitative comparison with the CO profiles of another model, namely the MCD (version 5.3), which introduces a new parameterization for non-condensable gases enrichment due to the condensation of $\mathrm{CO}_{2}$ (see Forget et al. (2008)). As can be seen from Figure 8, the MCD model simulates stronger vertical CO gradients than GEM in the polar region, especially close to the surface. However, in the equatorial region, the MCD model doesn't predict pronounced vertical gradient similar to the one that we retrieve. Based on these preliminary comparisons, it seems judicious to conclude that the vertical CO gradients observed are likely artificial and caused by the vertical sensitivity of PFS. Further work would be needed to confirm this. 


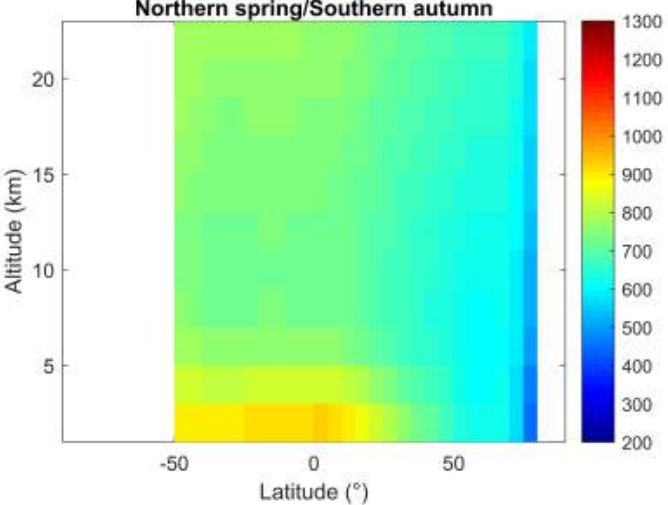

(a)

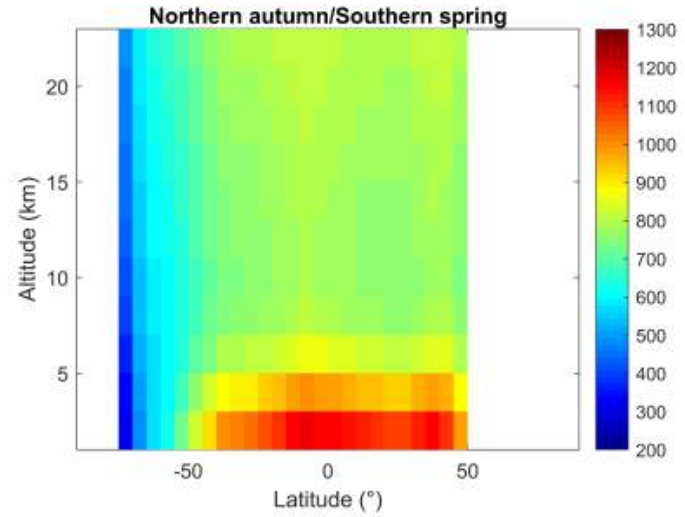

(c)

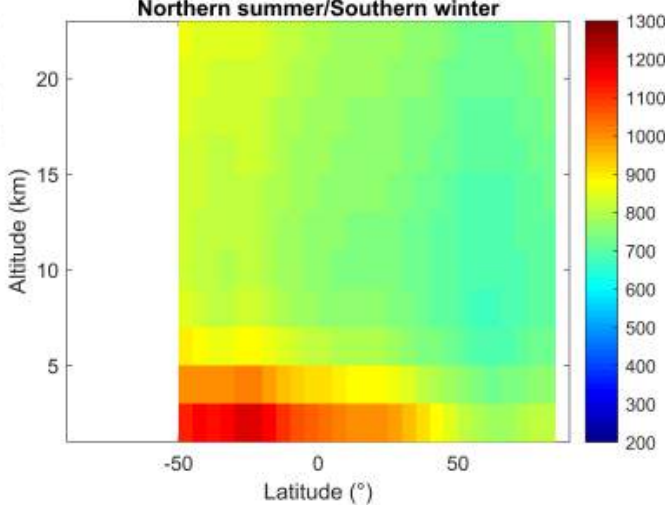

(b)

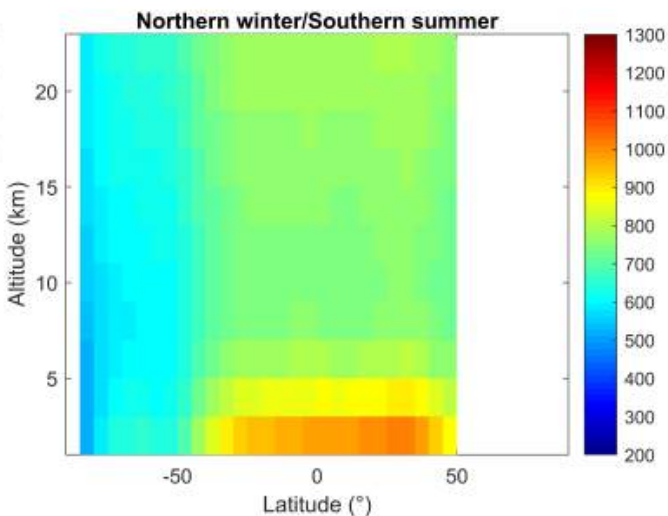

(d)

Figure 7. Vertical profile of CO VMR (color scale, ppm) retrieved from PFS spectra for each season (Northern spring $\mathrm{L}_{S}=\left[0-90^{\circ}\right]$, Northern summer $\mathrm{L}_{S}=\left[90-180^{\circ}\right]$, Northern autumn $\mathrm{L}_{S}=[180$ $\left.270^{\circ}\right]$, Northern winter $\left.\mathrm{L}_{S}=\left[270-360^{\circ}\right]\right)$. White areas indicate no retrievals. Note that a smoothing has been applied to the distributions, similarly as in Figure 3. 


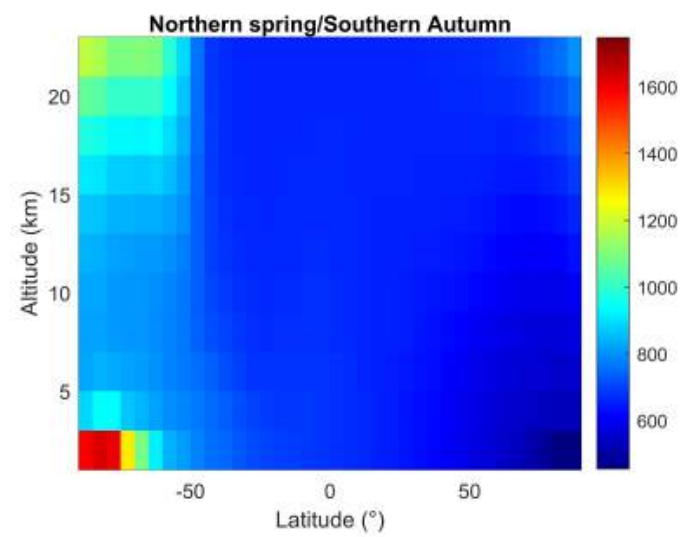

(a)

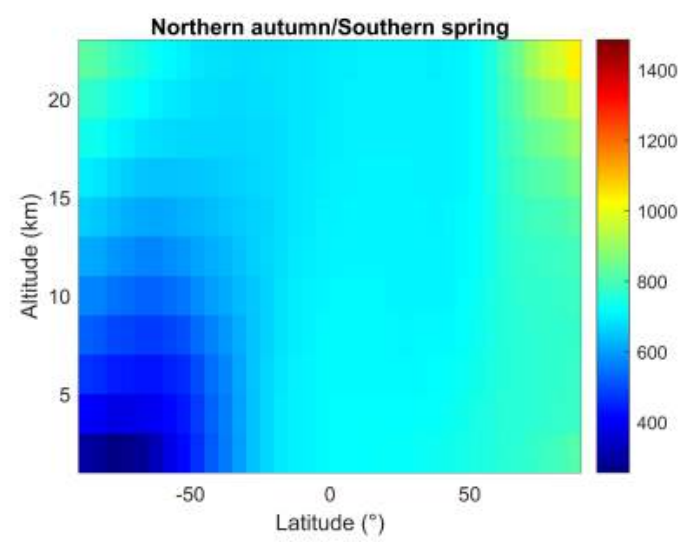

(c)

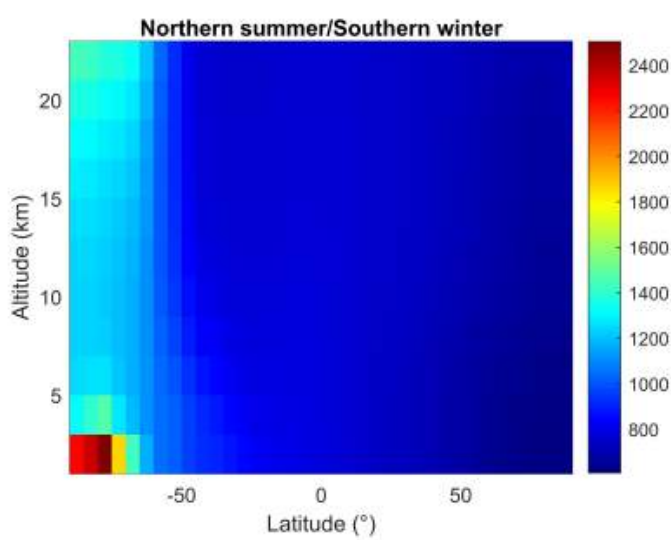

(b)

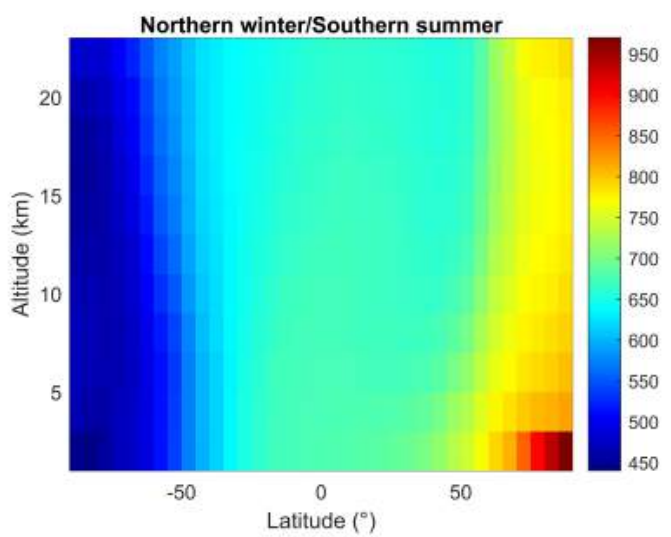

(d)

Figure 8. Vertical profile of CO VMR (color scale, ppm) simulated by the MCD model (version 5.3, Forget et al. (2008)) (Northern spring $\mathrm{L}_{S}=\left[0-90^{\circ}\right]$, Northern summer $\mathrm{L}_{S}=\left[90-180^{\circ}\right]$, Northern autumn $\mathrm{L}_{S}=\left[180-270^{\circ}\right]$, Northern winter $\left.\mathrm{L}_{S}=\left[270-360^{\circ}\right]\right)$. Note that the limits of the color bar are not the same as those in Figure 7.

\section{Conclusions}

A large subset of high spectral resolution measurements made by the PFS instrument onboard Mars Express have been inverted in the spectral region of the 1-0 fundamental band of $\mathrm{CO}$ (centered at $2143 \mathrm{~cm}^{-1}$ ), to reveal the seasonal cycle and the spatial distribution of $\mathrm{CO}$ in the Martian atmosphere in weak dust conditions (thermal infrared dust opacity lower than 0.2). Using the framework of the Optimal Estimation Method we have been able to retrieve weakly resolved $\mathrm{CO}$ profiles for more than 100000 individual PFS spectra. Considering the low information content on the $\mathrm{CO}$ vertical distribution the results have been discussed mainly in terms of a column-averaged CO VMR. Overall the total errors on individual CO columns are on average around 20-25\%.

On a global average, we found a mean CO VMR of about 820 ppm, with large variability around this mean value. Minimum values were retrieved in the polar regions (especially in the South pole, $\sim 400 \mathrm{ppm}$ against $600 \mathrm{ppm}$ in the North pole) during local spring/summer corresponding to the $\mathrm{CO}_{2}$ sublimation. At low latitudes, the $\mathrm{CO}$ column-averaged VMRs are less prone to seasonal variations and show variations of generally less than $100 \mathrm{ppm}$. However, we found a period of higher CO (up to $1000 \mathrm{ppm}$ ) around $\mathrm{L}_{S} \sim 160^{\circ}$. Our retrievals show good overall agreement with the latest CRISM climatology (M. D. Smith et 
al., 2018) in terms of amplitude of the seasonal cycle, with mean differences generally less than $100 \mathrm{ppm}$ in all latitude bands except the polar ones. At polar latitudes $\left(70^{\circ}-90^{\circ}\right)$ our retrieved values are typically larger than those of CRISM by 100 to $300 \mathrm{ppm}$, possibly because of the selection criteria applied in our case (which favours high CO). The PFS retrieved VMRs have also been compared to the latest GEM Mars model simulations. A satisfactory agreement was reported in terms of $\mathrm{CO}$ seasonal cycle with, however, a noticeable time mismatch at Southern low latitudes $\left(10^{\circ} \mathrm{S}-30^{\circ} \mathrm{S}\right.$ band) where the model simulates at the end of local autumn the increase in $\mathrm{CO}$ around $20^{\circ}$ of $\mathrm{L}_{S}$ earlier than what is measured by PFS. As already proposed by M. D. Smith et al. (2018), this time shift could be explained by transport time differences (different rate for the mixing of $\mathrm{CO}$ and the change of surface pressure). Also, our values were typically lower by 10 to $150 \mathrm{ppm}$, except for the latitude band $30^{\circ}-50^{\circ}$ for $\mathrm{L}_{S}=200^{\circ}-300^{\circ}$ where scattered values are retrieved in both hemispheres. This bias may be solved by lowering the initial CO VMR. In the current simulation, the initial conditions were chosen such to provide an optimal simulation of 3 datasets, and were not tuned to optimally reproduce the dataset presented here.

The spatial distribution of $\mathrm{CO}$ has been obtained for the four seasons. In terms of total columns they reveal mostly the variation in the topography (hence density). The distribution of the column-averaged VMR has allowed to depict the principal large-scale patterns of variations due to alternate $\mathrm{CO}_{2}$ condensation/sublimation cycle (CO depletion was found being more severe in the Southern hemisphere during local summer) but also several smaller-scale features, including enhancements around Alba Patera region (in the Northern spring) and around Noachis Terra (in the Southern summer).

Lastly, we have obtained and discussed the variations in the CO profiles in terms of latitude cross sections. Despite their low vertical resolution, the retrieved CO profiles showed systematic strong vertical gradient close to the surface in the mid-latitudes and equatorial regions. Preliminary comparisons with profiles simulated by the GEM-Mars model and MCD tend to suggest that these vertical features are likely artefacts related to the changing PFS vertical sensitivity.

\section{Acknowledgments}

This work is funded by the F.R.S. FNRS CRAMIC project under grant number T.0171.16. The PFS experiment was built at the Institute for Space Astrophysics and Planetology (formerly the Institute for Interplanetary Space Physics) of the National Institute for Astrophysics, and is currently funded by the Italian Space Agency (agreement number 20182-HH.0) in the context of the science activities for NOMAD-ExoMars and for PFS-MEx. Sophie Bauduin is Chargée de recherches with F.R.S.-FNRS. The authors would like to thank the LMD for providing the access to the Mars Climate Database, which was used to build the a priori vertical profile $\mathrm{CO}$ and the associated covariance matrix. CRISM and GEM model data are available through M. D. Smith et al. (2018) and Daerden et al. (2019), respectively. PFS data present in this work can be found in the general repository Open Science Framework: Bouche et al. (2020), https://osf.io/xsknv/?view_only=47d671dd84d84fe4b8802c726d293b4c.

\section{References}

Barret, B., Turquety, S., Hurtmans, D., Clerbaux, C., Hadji-Lazaro, J., Bey, I., ... Coheur, P.-F. (2005). Global carbon monoxide vertical distributions from spaceborne highresolution FTIR nadir measurements. Atmospheric chemistry and physics, 5(11), 2901-2914.

Billebaud, F., Brillet, J., Lellouch, E., Fouchet, T., Encrenaz, T., Cottini, V., ... Forget, F. (2009, October). Observations of CO in the atmosphere of Mars with PFS onboard Mars Express. Planetary and Space Science, 57, 1446-1457. doi: 10.1016/j.pss.2009 
.07 .004

Billebaud, F., Maillard, J., Lellouch, E., \& Encrenaz, T. (1992). The spectrum of Mars in the (1-0) vibrational band of CO. Astronomy and Astrophysics, 261, 647-657.

Billebaud, F., Rosenqvist, J., Lellouch, E., Maillard, J.-P., Encrenaz, T., \& Hourdin, F. (1998). Observations of CO in the atmosphere of Mars in the (2-0) vibrational band at 2.35 microns. Astronomy and Astrophysics, 333, 1092-1099.

Bouche, J., Bauduin, S., Giuranna, M., Robert, S., Aoki, S., Vandaele, A. C., ... Coheur, P.-F. (2019). Retrieval and characterization of carbon monoxide (CO) vertical profiles in the Martian atmosphere from observations of PFS/MEX. Journal of Quantitative Spectroscopy and Radiative Transfer.

Bouche, J., Coheur, P.-F., Giuranna, M., Wolkenberg, P., Nardi, L., Amoroso, M., ... Bauduin, S. (2020, Apr). Data: "Seasonal and spatial variability of carbon monoxide (CO) in the Martian atmosphere from PFS/MEX observations". OSF. Retrieved from osf.io/xsknv

Clancy, R. T., Muhleman, D. O., \& Berge, G. L. (1990). Global changes in the 0-70 km thermal structure of the Mars atmosphere derived from 1975 to 1989 microwave CO spectra. Journal of Geophysical Research: Solid Earth, 95(B9), 14543-14554.

Daerden, F., Neary, L., Viscardy, S., Muñoz, A. G., Clancy, R., Smith, M., .. Fedorova, A. (2019). Mars atmospheric chemistry simulations with the GEM-Mars general circulation model. Icarus, 326, 197-224.

Encrenaz, T., Fouchet, T., Melchiorri, R., Drossart, P., Gondet, B., Langevin, Y., ... Bézard, B. (2006). Seasonal variations of the Martian CO over Hellas as observed by OMEGA/Mars Express. Astronomy \& Astrophysics, 459(1), 265-270.

Forget, F., Millour, E., Montabone, L., \& Lefevre, F. (2008). Non condensable gas enrichment and depletion in the Martian polar regions. LPI Contrib, 1447, 9106.

Formisano, V., Angrilli, F., Arnold, G., Atreya, S., Bianchini, G., Biondi, D., ... others (2005). The Planetary Fourier Spectrometer (PFS) onboard the European Mars Express mission. Planetary and Space Science, 53(10), 963-974.

Garcia, D. (2010). Robust smoothing of gridded data in one and higher dimensions with missing values. Computational statistics \& data analysis, 54(4), 1167-1178.

Garcia, D. (2011). A fast all-in-one method for automated post-processing of PIV data. Experiments in fluids, 50(5), 1247-1259.

Giuranna, M., Formisano, V., Biondi, D., Ekonomov, A., Fonti, S., Grassi, D., ... others (2005). Calibration of the Planetary Fourier Spectrometer short wavelength channel. Planetary and Space Science, 53(10), 975-991.

Giuranna, M., Wolkenberg, P., Grassi, D., Aronica, A., Aoki, S., Scaccabarozzi, D., ... Formisano, V. (2019). The current weather and climate of Mars: 12 years of atmospheric monitoring by the Planetary Fourier Spectrometer on Mars Express. Icarus, 113406. doi: 10.1016/j.icarus.2019.113406

Haberle, R. M., Clancy, R. T., Forget, F., Smith, M. D., \& Zurek, R. W. (2017). The atmosphere and climate of Mars. Cambridge University Press.

Haberle, R. M., Forget, F., Colaprete, A., Schaeffer, J., Boynton, W. V., Kelly, N. J., \& Chamberlain, M. A. (2008). The effect of ground ice on the Martian seasonal $\mathrm{CO}_{2}$ cycle. Planetary and Space Science, 56(2), 251-255.

Holmes, J. A., Lewis, S. R., Patel, M. R., \& Smith, M. D. (2019). Global analysis and forecasts of carbon monoxide on Mars. Icarus.

Kelly, N. J., Boynton, W. V., Kerry, K., Hamara, D., Janes, D., Reedy, R., ... Haberle, R. M. (2006). Seasonal polar carbon dioxide frost on Mars: $\mathrm{CO}_{2}$ mass and columnar thickness distribution. Journal of Geophysical Research: Planets, 111(E3).

Kleinböhl, A., Friedson, A. J., \& Schofield, J. T. (2017). Two-dimensional radiative transfer for the retrieval of limb emission measurements in the Martian atmosphere. Journal of Quantitative Spectroscopy and Radiative Transfer, 187, 511-522.

Krasnopolsky, V. A. (2003). Spectroscopic mapping of Mars CO mixing ratio: Detection of north-south asymmetry. Journal of Geophysical Research: Planets, 108(E2). 
Lefèvre, F., \& Krasnopolsky, V. (2017). Atmospheric photochemistry. The Atmosphere and Climate of Mars, 18, 405.

Lellouch, E., Paubert, G., \& Encrenaz, T. (1991). Mapping of CO millimeter-wave lines in Mars' atmosphere: The spatial variability of carbon monoxide on Mars. Planetary and Space Science, 39(1-2), 219-224.

Lian, Y., Richardson, M. I., Newman, C. E., Lee, C., Toigo, A. D., Mischna, M. A., \& Campin, J.-M. (2012). The Ashima/MIT Mars GCM and argon in the martian atmosphere. Icarus, 218(2), 1043-1070.

Neary, L., \& Daerden, F. (2018). The GEM-Mars general circulation model for Mars: Description and evaluation. Icarus, 300, 458-476. doi: 10.1016/j.icarus.2017.09.028

Nelli, S. M., Murphy, J. R., Sprague, A. L., Boynton, W. V., Kerry, K. E., Janes, D. M., \& Metzger, A. E. (2007). Dissecting the polar dichotomy of the noncondensable gas enhancement on Mars using the NASA Ames Mars General Circulation Model. Journal of Geophysical Research: Planets, 112(E8).

Rodgers, C. D. (2000). Inverse methods for atmospheric sounding: theory and practice (Vol. 2). World scientific.

Seviour, W. J., Waugh, D. W., \& Scott, R. K. (2017). The stability of Mars's annular polar vortex. Journal of the Atmospheric Sciences, 74 (5), 1533-1547.

Sindoni, G., Formisano, V., \& Geminale, A. (2011). Observations of water vapour and carbon monoxide in the Martian atmosphere with the SWC of PFS/MEX. Planetary and Space Science, 59(2-3), 149-162. doi: 10.1016/j.pss.2010.12.006

Smith, D. E., Zuber, M. T., Frey, H. V., Garvin, J. B., Head, J. W., Muhleman, D. O., ... others (2001). Mars Orbiter Laser Altimeter: Experiment summary after the first year of global mapping of Mars. Journal of Geophysical Research: Planets, 106(E10), 23689-23722.

Smith, M. D., Daerden, F., Neary, L., \& Khayat, A. (2018). The climatology of carbon monoxide and water vapor on Mars as observed by CRISM and modeled by the GEMMars general circulation model. Icarus, 301, 117-131. doi: 10.1016/j.icarus.2017.09 .027

Smith, M. D., Wolff, M. J., Clancy, R. T., \& Murchie, S. L. (2009). Compact Reconnaissance Imaging Spectrometer observations of water vapor and carbon monoxide. Journal of Geophysical Research: Planets, 114(E2). doi: 10.1029/2008JE003288

Sprague, A., Boynton, W. V., Kerry, K., Janes, D., Hunten, D., Kim, K., ... Metzger, A. (2004). Mars' south polar Ar enhancement: A tracer for south polar seasonal meridional mixing. Science, 306(5700), 1364-1367.

Vandaele, A. C., Lopez-Moreno, J.-J., Patel, M. R., Bellucci, G., Daerden, F., Ristic, B., ... others (2018). NOMAD, an integrated suite of three spectrometers for the ExoMars trace gas mission: technical description, science objectives and expected performance. Space Science Reviews, $214(5), 80$.

Wolkenberg, P., Giuranna, M., Grassi, D., Aronica, A., Aoki, S., Scaccabarozzi, D., \& Saggin, B. (2018). Characterization of dust activity on Mars from MY27 to MY32 by PFS-MEX observations. Icarus, 310, 32-47. doi: 10.1016/j.icarus.2017.10.045 